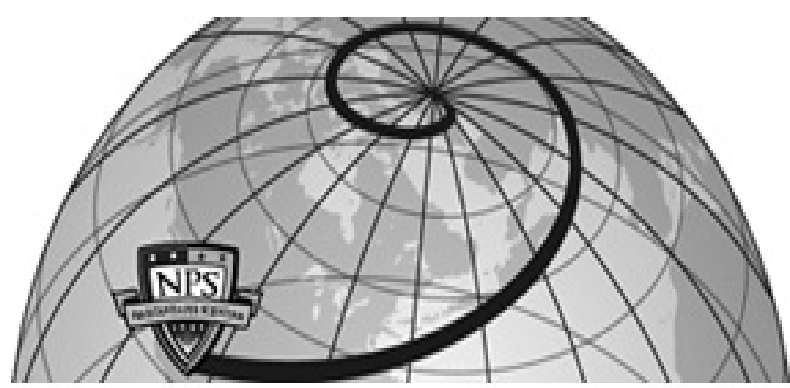

Calhoun: The NPS Institutional Archive DSpace Repository

On the design of LPM address generators using multiple LUT Cascades on FPGAs

Qin, Hui; Sasao, Tsutomu; Butler, Jon T.

H. Qin, T. Sasao, and J. T. Butler, "On the design of LPM address generators using multiple LUT Cascades on FPGAs," International Journal of Electronics, Vol. 94, Issue 5, May 2007, pp.451-467, https://hdl.handle.net/10945/35851

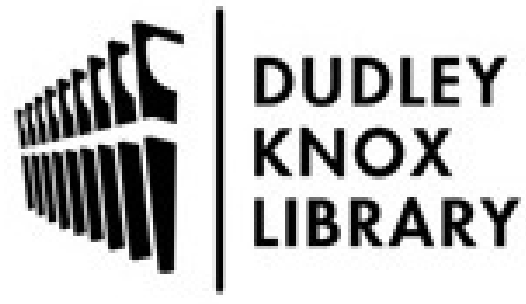

http://www.nps.edu/library
Calhoun is the Naval Postgraduate School's public access digital repository for research materials and institutional publications created by the NPS community. Calhoun is named for Professor of Mathematics Guy K. Calhoun, NPS's first appointed -- and published -- scholarly author.

Dudley Knox Library / Naval Postgraduate School 411 Dyer Road / 1 University Circle Monterey, California USA 93943 


\title{
On the Design of LPM Address Generators Using Multiple LUT Cascades on FPGAs
}

\author{
Hui Qin ${ }^{\dagger a)}$, Tsutomu Sasao ${ }^{\dagger b)}$, and Jon T. Butler ${ }^{\ddagger c)}$ \\ $\dagger$ Department of Computer Science and Electronics, Kyushu Institute of Technology, \\ 680-4, Kawazu, Iizuka, Fukuoka, 820-8502, Japan \\ $\ddagger$ Department of Electrical and Computer Engineering, Naval Postgraduate School, \\ Code EC/Bu, Monterey, CA 93943-5121 \\ (Received November 26, 2006)
}

\begin{abstract}
We propose the multiple LUT cascade as a means to configure an $n$-input LPM (Longest Prefix Match) address generator commonly used in routers to determine the output port given an address. The LPM address generator accepts $n$-bit addresses which it matches against $k$ stored prefixes. We implement our design on a Xilinx Spartan-3 FPGA for $n=32$ and $k=504 \sim 511$. Also, we compare our design to a Xilinx proprietary TCAM (ternary content-addressable memory) design and to another design we propose as a likely solution to this problem. Our best multiple LUT cascade implementation has 5.17 times more throughput, 40.71 times more throughput/area and is 2.97 times more efficient in terms of area-delay product than Xilinx's proprietary design, but its area is only $15 \%$ of Xilinx's design. Furthermore, we derive a method to determine the optimum configuration of the multiple LUT cascade on an FPGA.
\end{abstract}

Keywords: LPM address generator; Multiple LUT cascade; FPGA

\section{Introduction}

The need for higher internet speeds is likely to be the subject of intense interest for many years to come. A network's speed is directly related to the speed with which a node can switch a packet from an input port to an output port. This, in turn, depends on how fast a packet's address can be accessed in memory. The longest prefix match (LPM) problem is one of determining the output port address from a list of prefix vectors stored in memory. For example, if the prefix vector $01001^{* * * *}$ is stored in memory, then the packet address 010011111 matches this entry. That is, each bit in the packet address matches

Email: ${ }^{a)}$ qinhui@aries01.cse.kyutech.ac.jp; ${ }^{b)}$ sasao@cse.kyutech.ac.jp; ${ }^{c)}$ jon_butler@msn.com

International Journal of Electronics ISSN 0020-7217 print/ ISSN 1362-3060 online (C2006 Taylor \& Francis Ltd

http://www.tandf.co.uk/journals

DOI: $10.1080 / 00207210 \times x \times x \times x \times x \times x x x x$ 
exactly the corresponding bit in the prefix vector or there is a ${ }^{*}$ or don't care in that position. If other stored prefixes match the packet address, then the prefix with the least don't care values determines the output port address. That is, the memory entry corresponding to the longest prefix match determines the output port.

An ideal device for this application is a ternary content-addressable memory (TCAM) (Pagiamtzis et al. 2006, Song et al. 2005). The descriptor "ternary" refers to the three values stored, 0, 1, and *. In (Kasnavi et al. 2005), the authors proposed pipelined TCAMs for the longest prefix match to increase TCAM efficiency. In (Wang et al. 2005), the authors used a TCAM and a small DRAM for the longest prefix match to reduce the required size of TCAM. Unfortunately, TCAM still dissipates more power than standard RAM (Renesas 2005).

Several authors have proposed the use of standard RAM in LPM design. Gupta, Lin, and McKeown showed a mechanism to perform LPM every memory access (Gupta et al. 1998). Dharmapurikar, Krishnamurthy, and Taylor have proposed the use of Bloom filters to solve the LPM problem (Dharmapurikar et al. 2003). Sasao and Butler have shown that a fast, power-efficient TCAM realization using a look-up table (LUT) cascade (Sasao et al. 2006).

In this paper, we propose an extension to the LUT cascade realization: a multiple LUT cascade realization that consists of multiple LUT cascades connected to a special encoder. This offers even more efficient realizations in an architecture that is more easily reconfigured when additional prefix vectors are placed in the prefix table.

We have implemented six LPM address generators on the Xilinx Spartan-3 FPGA (XC3S4000-5): Four using multiple LUT cascades, one using Xilinx's TCAM realization based on the Xilinx IP core, and one using registers and gates. In addition, we compare the six LPM address generators on the basis of delay, delay-area product, throughput, throughput/area, and FPGA resources used.

A preliminary version of this paper was presented at ARC2006 (Qin et al. 2006). We extend these results by introducing the optimum configuration of the multiple LUT cascade, and by showing how to realize the optimum multiple LUT cascade on an FPGA.

The rest of the paper is organized as follows: Section 2 describes the multiple LUT cascade. Section 3 shows other realizations for the LPM address generators. Section 4 presents the implementations of the LPM address generator using an FPGA. Section 5 shows the experimental results. Section 6 discusses the optimum configuration of the multiple LUT cascade implemented on an FPGA. And finally, Section 7 concludes the paper. 


Table 1. LPM table.
\begin{tabular}{|c|c|}
\hline Address & Prefix Vector \\
\hline 1 & 1000 \\
\hline 2 & $010^{*}$ \\
\hline 3 & $01^{* *}$ \\
\hline 4 & $1^{* * *}$ \\
\hline 5 & $0^{* * *}$ \\
\hline
\end{tabular}

Table 2. LPM function.
\begin{tabular}{|c|c||c|c|}
\hline Input & $\begin{array}{c}\text { Output } \\
\text { Address }\end{array}$ & Input & $\begin{array}{c}\text { Output } \\
\text { Address }\end{array}$ \\
\hline 0000 & 5 & 1000 & 1 \\
\hline 0001 & 5 & 1001 & 4 \\
\hline 0010 & 5 & 1010 & 4 \\
\hline 0011 & 5 & 1011 & 4 \\
\hline 0100 & 2 & 1100 & 4 \\
\hline 0101 & 2 & 1101 & 4 \\
\hline 0110 & 3 & 1110 & 4 \\
\hline 0111 & 3 & 1111 & 4 \\
\hline
\end{tabular}

\section{Multiple LUT cascades}

\section{$2.1 \quad L P M$ address generators}

A content-addressable memory (CAM) (Shafai et al. 1998) stores 0's and 1's and produces the address of the given data. A TCAM, unlike a CAM, stores 0 's, 1's, and ${ }^{*}$ 's, where ${ }^{*}$ is a don't care value that matches both 0 and 1 .

TCAMs are extensively used in routing tables for the internet. A routing table specifies an interface identifier corresponding to the longest prefix that matches an incoming packet, in a process called Longest Prefix Match (LPM). In the LPM table, the ternary vectors have restricted patterns: the prefix consists of only 0's and 1's, and the postfix consists of only *'s (don't cares). In this paper, this type of vector is called a prefix vector.

Definition 2.1 An $n$-input $m$-output $k$-entry LPM table stores $k n$-element prefix vectors. To assure that the longest prefix address is produced, TCAM entries are stored in descending prefix length, and the first match starting from the top of the table determines the LPM table's output. An address is an melement binary vector for $m=\left\lceil\log _{2}(k+1)\right\rceil$, where $\lceil a\rceil$ denotes the smallest integer greater than or equal to $a$. The corresponding LPM function is a logic function $f: B^{n} \rightarrow B^{m}$, where $f(\vec{x})$ is the smallest address of an entry that is identical to $\vec{x}$ except possibly for don't care values. If no such entry exists, $f(\vec{x})=0^{m}$. The LPM address generator is a circuit that realizes the LPM function.

Example 2.2 Table 1 shows an LPM table with 5 4-element prefix vectors. Table 2 shows the corresponding LPM function. It has 16 entries, one for each 4-bit input. The output address is stored for each input corresponding to the address of the longest prefix vector that matches it.

\subsection{An LUT cascade realization of LPM address generators}

An LPM function, such as that shown in Table 2, can be realized by a single memory which operates as a programmable combinational logic circuit. 
However, this often requires prohibitively large memory size.

THEOREM 2.3 (Sasao 2006) An n-input LPM address generator with $k$ prefix vectors can be realized by an LUT cascade, where each cell realizes a p-input, $r$-output combinational logic function. Let $s$ be the necessary number of levels or cells. Then,

$$
s \leq\left\lceil\frac{n-r}{p-r}\right\rceil
$$

where $p>r$ and $r=\left\lceil\log _{2}(k+1)\right\rceil$.

\subsection{LPM address generators using the multiple LUT cascade}

A single LUT cascade realization of an LPM function often requires many levels. Since the delay is proportional to the number of levels in a cascade, we wish to reduce the number of levels. According to (1), if we increase $p$, the number of inputs to each cell, then the number of levels $s$ is reduced. For each increase by 1 of $p$, the memory needed to realize the cell is doubled. However, as shown in Figure 1, we can use a multiple LUT cascade to reduce the number of levels $s$ while keeping $p$ fixed. For an $n$-input LPM function with $k$ prefix vectors, let the number of rails of each LUT cascade be $r$. First, starting at the top of the LPM table, partition the set of prefix vectors into $g$ groups of $2^{r}-1$ vectors each, except the last group, which has $2^{r}-1$ or fewer vectors, where $g=\left\lceil\frac{k}{2^{r}-1}\right\rceil$. For each group of prefix vectors, form an independent LPM function. Next, partition the set of $n$ inputs into $s$ groups. The inputs within a group will apply to a single cell within each cascade. Then, realize each LPM function by an LUT cascade. Thus, we need a total of $g$ LUT cascades, where each LUT cascade consists of $s$ cells. Finally, use a special encoder to produce the LPM address. Let $v_{i}(i=1,2, \ldots, g)$ be the $i$-th input of the special encoder from the $i$-th LUT cascade, and let $v_{\text {out }}$ be the output value of the special encoder. That is, $v_{i}$ is the output value of the $i$-th LUT cascade, where its binary output values are viewed as a standard binary number. Similarly, $v_{\text {out }}$ is the output of the special encoder, where its binary output values are viewed as a standard binary number. Then, we have the relation:

$$
v_{\text {out }}= \begin{cases}v_{i}+(i-1)\left(2^{r}-1\right) & \text { if } v_{i} \neq 0 \text { and } v_{j}=0 \text { for all } 1 \leq j \leq i-1 \\ 0 & \text { if } v_{i}=0 \text { for all } 1 \leq i \leq g\end{cases}
$$

Note that $v_{\text {out }}$ is the position of a prefix vector $v$ in the complete LPM table, while $i$ is the index to the LUT cascade storing $v \cdot(i-1)\left(2^{r}-1\right)$ is the position in the LPM table of the last entry of the previous $(i-1)$-th LUT cascade or 
is 0 in the case of the first LUT cascade. Adding $v_{i}$ to this yields the position of $v$ in the complete LPM table.

Example 2.4 Consider an $n$-input LPM function with $k$ prefix vectors. When $k=1000$ and $n=32$, by Theorem 2.3 , we have $r=10$. Let $p=r+1=11$. When we use a single LUT cascade to realize the function, by Theorem 2.3, we need $\left\lceil\frac{n-r}{p-r}\right\rceil=22$ cells, and the number of levels of the LUT cascade is also 22. Since each cell has 11 address lines and 10 outputs, the total memory size needed to realize the cascade is $2^{11} \times 10 \times 22=450,560$ bits. Note that the memory size of each cell, $2^{11} \times 10=20,480$ bits, is too large to be realized by a single block_RAM (BRAM) of our FPGA, which stores 18, 432 bits.

However, if we use a multiple LUT cascade to realize the function, we can reduce the number of levels and the total memory. Also, the cells will fit into the BRAMs in the FPGAs. Partition the set of vectors into two groups, and realize each group independently; this requires two LUT cascades. For each LUT cascade, the number of vectors is 500, so we have $r=9$. Also, let $p=r+2=11$. Then, we need $\left\lceil\frac{n-r}{p-r}\right\rceil=12$ cells in each cascade. Note that the number of levels of the LUT cascades is 12, which is smaller than the 22 needed in the single LUT cascade realization. Since each cell consists of a memory with 9 outputs and at most 11 address lines, the total memory size is at most $2^{11} \times 9 \times 12 \times 2=442,368$ bits. Also, note that the size of the memory for a single cell is $2^{11} \times 9=18,432$ bits. This fits exactly in the BRAMs of the FPGAs.

Thus, the multiple LUT cascade not only reduces the number of levels and the total memory, but also reduces the size of cells to fit into the available memory in the FPGAs.

Fig. 1 shows the multiple LUT cascade realization. It consists of multiple LUT cascades and a special encoder. The inputs of each LUT cascade are common with other LUT cascades, while the outputs of each LUT cascade are connected to the special encoder. Each LUT cascade realizes an LPM function, while the special encoder generates the LPM address from the outputs of cascades.

The detailed design of each LUT cascade is shown in Fig. 2. Here $\vec{x}_{i}(i=$ $1,2, \ldots, s)$ denotes the primary inputs to the $i$-th cell, $\vec{d}_{i}(i=1,2, \ldots, s)$ denotes the data inputs to the $i$-th cell and provides the data value to be written in the RAM of the $i$-th cell, $r$ denotes the number of rails, where $r \leq\left\lceil\log _{2}\left(\frac{k}{g}+1\right)\right\rceil$, $\vec{c}_{j}(j=2,3, \ldots, s)$ denotes the additional inputs to the $j$-th cell and is used to select the RAM location along with $\vec{x}_{j}$ for write access. Note that $\vec{c}_{j}$ and $\vec{d}_{i}$ are represented by $r$ bits. All RAMs except perhaps the last one have $p$ address lines; the last RAM has at most $p$ address lines. When $W E$ is high, $\vec{c}_{j}$ is connected to the RAM through a MUX, allowing data to be written into 


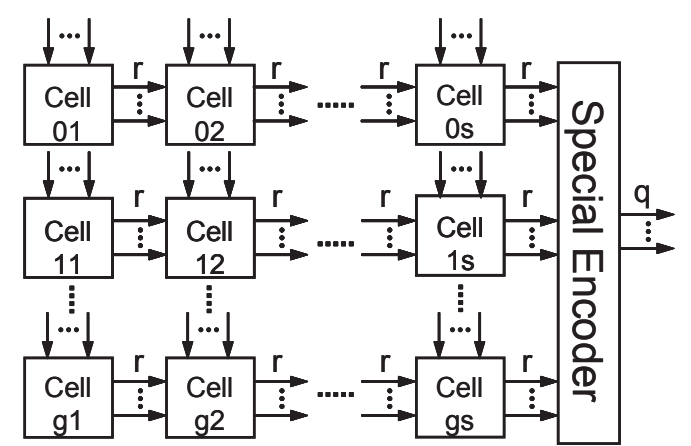

Figure 1. Architecture of the multiple LUT cascade.

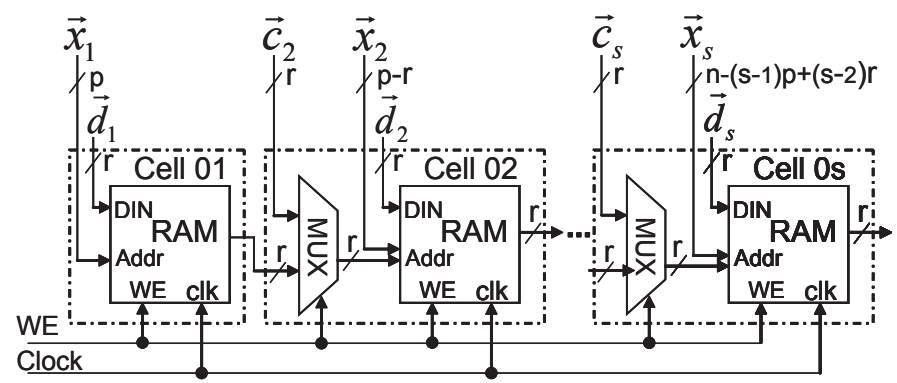

Figure 2. Detailed design of the LUT cascade.

Table 3. 6-entry LPM table.

\begin{tabular}{|c|c|}
\hline Address & Prefix Vector \\
\hline 1 & 100000 \\
\hline 2 & $10010^{*}$ \\
\hline 3 & $1010^{* *}$ \\
\hline \hline 4 & $101^{* * *}$ \\
\hline 5 & $10^{* * * *}$ \\
\hline 6 & $1^{* * * * *}$ \\
\hline
\end{tabular}

Table 4. Truth table for the corresponding LPM function.
\begin{tabular}{|c|c|c|c|c|c|c|c|c|c|}
\hline \multicolumn{7}{|c|}{ Input } & \multicolumn{5}{c|}{ Output } & LUT \\
$x_{1}$ & $x_{2}$ & $x_{3}$ & $x_{4}$ & $x_{5}$ & $x_{6}$ & out $_{2}$ & out $_{1}$ & out $_{0}$ & Cascade \\
\hline 1 & 0 & 0 & 0 & 0 & 0 & 0 & 0 & 1 & Upper \\
1 & 0 & 0 & 1 & 0 & $*$ & 0 & 1 & 0 & Cells 1 \\
1 & 0 & 1 & 0 & $*$ & $*$ & 0 & 1 & 1 & and 2 \\
\hline 1 & 0 & 1 & 1 & $*$ & $*$ & 1 & 0 & 0 & Lower \\
1 & 0 & 0 & $*$ & $*$ & $*$ & 1 & 0 & 1 & Cells 3 \\
1 & 1 & $*$ & $*$ & $*$ & $*$ & 1 & 1 & 0 & and 4 \\
\hline
\end{tabular}

the RAMs. When $W E$ is low, the outputs of the RAMs are connected to the inputs of the succeeding RAMs through a MUX, and the circuit is a cascade that realizes the LPM function. Note that the RAMs are synchronous RAMs. Therefore, the LUT cascade resembles a shift register.

Example 2.5 Table 3 shows a 6-input 3-output 6-entry LPM table, and the truth table of the corresponding LPM function is shown in Table 4. Note that the entries in the two tables are similar. Table 4 is a compact truth table, showing only non-zero outputs. Its input combinations must be disjoint. Thus, the two tables are the same except for three entries. 


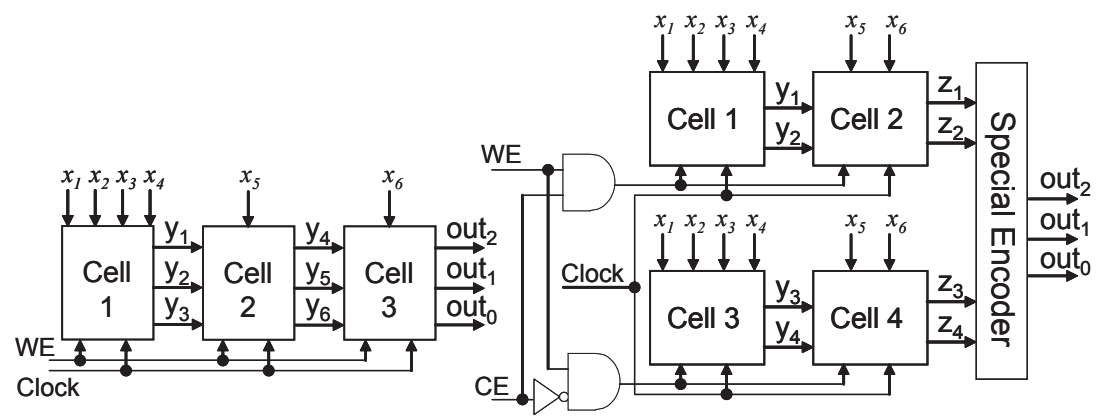

(a) Single LUT cascade realization

(b) Multiple LUT cascade realization

Figure 3. Single LUT cascade realization and the multiple LUT cascade realization.

Single Memory Realization: The number of address lines is 6, and the number of outputs is 3 . Thus, the total amount of memory is $2^{6} \times 3=192$ bits.

Single LUT Cascade Realization: Since there are $k=6$ prefix vectors, by Theorem 2.3, the number of rails is $r=\left\lceil\log _{2}(6+1)\right\rceil=3$. Let the number of address lines for the memory in a cell be $p=4$. By partitioning the inputs into three disjoint sets $\left\{x_{1}, x_{2}, x_{3}, x_{4}\right\},\left\{x_{5}\right\}$, and $\left\{x_{6}\right\}$, we have the cascade in Fig. 3 (a). For simplicity, only the signal lines for the cascade realization are shown. Other lines, such as for storing data, are omitted.

The total amount of memory is $2^{4} \times 3 \times 3=144$ bits, and the number of levels is $s=3$. Note that the single LUT cascade requires $75 \%$ of the memory needed in the single memory realization.

Multiple LUT Cascade Realization: Partition Table 3 into two parts, each with three prefix vectors. The number of rails in the LUT cascades associated with each separate LPM table is $\left\lceil\log _{2}(3+1)\right\rceil=2$. Let the number of address lines for the memory in a cell be $p=4$. By partitioning the inputs into two disjoint sets $\left\{x_{1}, x_{2}, x_{3}, x_{4}\right\}$ and $\left\{x_{5}, x_{6}\right\}$, we obtain the realization in Fig. 3 (b). The upper LUT cascade realizes the upper part of the Table 4, while the lower LUT cascade realizes the lower part of the Table 4 . The contents of each cell is shown in Table 5 .

Let $v_{1}$ be the output value of the upper LUT cascade, let $v_{2}$ be the output value of the lower LUT cascade, and let $v_{\text {out }}$ be the output value of the special encoder. Then, in Table $5,\left(z_{1}, z_{2}\right)$ viewed as a standard binary number, has value $v_{1}$, while $\left(z_{3}, z_{4}\right)$ viewed as a standard binary number, has value $v_{2}$. The special encoder generates the LPM address from the pair of outputs, $\left(z_{1}, z_{2}\right)$ and $\left(z_{3}, z_{4}\right)$ :

$$
\text { out }_{2}=\bar{z}_{1} \bar{z}_{2}\left(z_{3} \vee z_{4}\right)
$$


Table 5. Truth tables for the cells in the multiple LUT cascade realization.

\begin{tabular}{|c|c|c|c|c|c|c|c|c|c|c|c|c|c|c|c|c|c|c|c|c|c|c|c|}
\hline \multicolumn{12}{|c|}{ Cell 1 and Cell 2 (upper LUT cascade) } & \multicolumn{12}{|c|}{ Cell 3 and Cell 4 (lower LUT cascade) } \\
\hline$x_{1}$ & $x_{2}$ & $x_{3}$ & $x_{4}$ & $y_{1}$ & $y_{2}$ & $x_{5}$ & $x_{6}$ & $z_{1}$ & $z_{2}$ & $v_{1}$ & $v_{\text {out }}$ & $x_{1}$ & $x_{2}$ & $x_{3}$ & $x_{4}$ & $y_{3}$ & $y_{4}$ & $x_{5}$ & $x_{6}$ & $z_{3}$ & $z_{4}$ & $v_{2}$ & $v_{\text {out }}$ \\
\hline 1 & 0 & 0 & 0 & 0 & 0 & 0 & 0 & 0 & 1 & 1 & 001 & 1 & 0 & 1 & 1 & 0 & 0 & $*$ & $*$ & 0 & 1 & 1 & 100 \\
\hline 1 & 0 & 0 & 1 & 0 & 1 & $\overline{0}$ & * & 1 & 0 & 2 & 010 & 1 & 0 & 0 & * & 0 & 1 & $*$ & $*$ & 1 & 0 & 2 & 101 \\
\hline 1 & 0 & 1 & 0 & 1 & 0 & $*$ & $*$ & 1 & 1 & 3 & 011 & 1 & 1 & $*$ & $*$ & 1 & 0 & $*$ & $*$ & 1 & 1 & 3 & 110 \\
\hline \multicolumn{4}{|c|}{ Other values } & 1 & 1 & $*$ & * & 0 & 0 & 0 & $\dagger$ & \multicolumn{4}{|c|}{ Other values } & 1 & 1 & $*$ & $*$ & 0 & 0 & 0 & $\bar{\dagger}$ \\
\hline & & & & & her & val & & 0 & 0 & 0 & $\dagger$ & & & & & \multicolumn{4}{|c|}{ Other values } & 0 & 0 & 0 & $t$ \\
\hline
\end{tabular}

$\dagger$ depends on values from the other LUT cascade.

$$
\begin{aligned}
& \text { out }_{1}=z_{1} \vee \bar{z}_{2} z_{3} z_{4}, \\
& \text { out }_{0}=z_{2} \vee \bar{z}_{1} z_{3} \bar{z}_{4} .
\end{aligned}
$$

Note that $\left(\right.$ out $_{2}$, out $_{1}$, out $\left._{0}\right)$ viewed as a standard binary number, has value $v_{\text {out }}$ corresponding to the address in Table 3 . The total memory size is $2^{4} \times 2 \times 4=$ $128 \mathrm{bits}$, and the number of levels is 2 . Note that the multiple LUT cascade realization requires $89 \%$ of the memory and one fewer level than the single LUT cascade realization.

\section{Other realizations}

\subsection{Xilinx's TCAM}

Xilinx (Website of Xilinx) provides a proprietary realization of a TCAM that is produced by the Xilinx CORE Generator tool. Since a TCAM can directly realize an LPM address generator, we compare our proposed multiple LUT cascade realization with Xilinx's TCAM. In the Xilinx CORE Generator 7.1i, we used the following parameters to produce TCAMs.

- Implementation: SRL16.

- Mode: Standard ternary mode to generate a standard ternary CAM.

- Depth: $k$, the number of words or vectors stored in the TCAM.

- Data width: $n$, the number of bits in words or vectors.

- Match Address Type: Binary encoded.

- Address Resolution: Lowest.

\subsection{Registers and gates}

We also compare our proposed multiple LUT cascade realization with a direct realization using registers and gates, as shown in Fig 4. We use a register pair (Reg. 1 and Reg. 0) to store each digit of a ternary vector. For example, if the digit is * (don't care), the register pair stores $(1,1)$. Thus, for $n$ bit data, we need a $2 n$-bit register. The comparison circuit consists of an $n$-input AND 


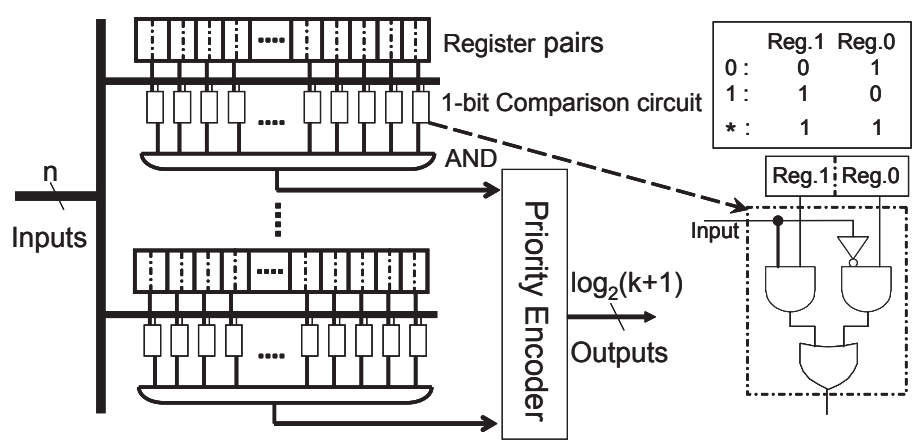

Figure 4. Realization of the address generator with registers and gates.

gate and $n$ 1-bit comparison circuits, each of which produces a 1 if and only if the input bit matches the stored bit or the stored bit is don't care (* or 11).

For each prefix vector of an $n$-input LPM address generator, we need a $2 n$ bit register, $n$ 1-bit comparison circuits, and an $n$-input AND gate. For an $n$-input address generator with $k$ registered prefix vectors, we need $k 2 n$-bit registers, $n k 1$-bit comparison circuits, and $k n$-input AND gates. In addition, we need a priority encoder with $k$ inputs and $\left\lceil\log _{2}(k+1)\right\rceil$ outputs to generate the LPM address. If the $n$-input AND gate is realized as a cascade of 2 -input AND gates, this circuit can be considered as a special case of the multiple LUT cascade architecture, where $r=1, p=2$, and $g=k$. Note that the output encoder circuit is a standard priority encoder.

\section{$4 \quad$ FPGA implementations}

We implemented the LPM address generators for 32 inputs and 504 511 registered prefix vectors on Xilinx Spartan-3 FPGAs (XC3S4000-5) in three ways, the multiple LUT cascade, Xilinx CORE Generator 7.1i, and registers and gates. XC3S4000-5 (Spartan-3 FPGA data sheet 2005) has 96 BRAMs and 27,648 slices. Each BRAM contains $18 \mathrm{~K}$ bits, and each slice consists of two 4-input LUTs, two D-type flip-flops, and multiplexers. For each implementation, we described the circuit by Verilog HDL, and then used Xilinx ISE 7.1i to synthesize and to perform place and route.

First, we used the multiple LUT cascade to realize the LPM address generators. To use the BRAMs in the FPGA efficiently, we chose the memory size of a cell in the LUT cascade not to exceed the size of a BRAM unit. Let $p$ be number of address lines of the memory in the cell. Since each BRAM contains $2^{11} \times 9$ bits, we have the relation: $2^{p} \cdot r \leq 2^{11} \times 9$, where $r$ is the number of rails. Thus, we have $p=\left\lfloor\log _{2}(9 / r)\right\rfloor+11$, where $\lfloor a\rfloor$ denotes the largest integer less than or equal to $a$. 
Table 6. Four multiple LUT cascade realizations.

\begin{tabular}{|c|c|c|c|c|c|}
\hline Design & Number of prefix vectors & $\boldsymbol{r}$ & $p$ & Group & Level \\
\hline$r 6 p 11$ & 504 & 6 & 11 & 8 & 6 \\
\hline$r 7 p 11$ & 508 & 7 & 11 & 4 & 7 \\
\hline$r 8 p 11$ & 510 & 8 & 11 & 2 & 8 \\
\hline$r 9 p 11$ & 511 & 9 & 11 & 1 & 12 \\
\hline
\end{tabular}

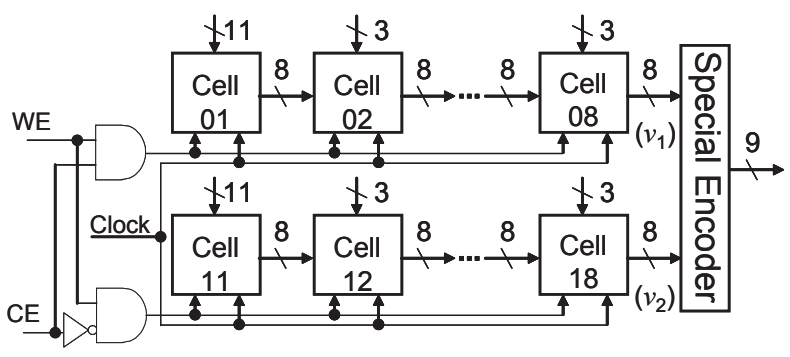

Figure 5. Realization of $r 8 p 11$.

We designed four LPM address generators $r 6 p 11, r 7 p 11, r 8 p 11$, and $r 9 p 11$, as shown in Table 6, where the column Number of prefix vectors denotes the number of registered prefix vectors, the column $\boldsymbol{r}$ denotes the number of rails, the column $\boldsymbol{p}$ denotes the number of address lines of the RAM in a cell, the column Group denotes the number of LUT cascades, and the column Level denotes the number of levels or cells in the LUT cascade.

To explain Table 6, consider $r 8 p 11$ which is shown in Fig 5 . For $r 8 p 11$, since the number of rails is $r=8$, the number of groups is $\left\lceil\frac{510}{2^{8}-1}\right\rceil=2$. Thus, we need two LUT cascades. Since each LUT cascade consists of 8 cells, the number of levels of $r 8 p 11$ is 8 . To efficiently use BRAMs in the FPGA, the number of address lines of the RAM in the cell is set to $p=\left\lfloor\log _{2}(9 / 8)\right\rfloor+11=11$. Let $v_{1}$ be the value of the outputs of the upper LUT cascade, let $v_{2}$ be the value of the outputs of the lower LUT cascade, and let $v_{\text {out }}$ be the value of the outputs of the special encoder. Then, we have the relation:

$$
v_{\text {out }}= \begin{cases}v_{2}+255 & \text { if } v_{1}=0 \text { and } v_{2} \neq 0 \\ v_{1} & \text { otherwise }\end{cases}
$$

The circuit realizing this expression requires 11 slices on the FPGA. For the whole circuit, $r 8 p 11$ requires 16 BRAMs and 69 slices. From this table, we can see that decreasing $r$, increases the number of groups, but decreases the number of levels.

Next, we used the Xilinx CORE Generator 7.1i tool to produce Xilinx's TCAM. Since the Xilinx CORE Generator 7.1i does not support TCAMs with 
32 inputs and 505 511 registered prefix vectors, we designed a TCAM with 32 inputs and 504 registered prefix vectors. The resulting TCAM required 8,590 slices. Note that Xilinx's TCAM requires one clock cycle to find a match.

Finally, we designed the LPM address generator with $n=32$ inputs and $k=511$ registered prefix vectors using registers and gates, as shown in Fig 4 . This design is denoted Reg-Gates. Note that the number of inputs is 32 and the number of outputs is 9 . This design required 27,646 slices.

\section{Performance and comparisons}

In Table 7, we show the performance of multiple LUT cascade realizations (i.e., $r 6 p 11, r 7 p 11, r 8 p 11$, and $r 9 p 11$ ), and compare them with Xilinx's TCAM and Reg-Gates. In Table 7, the column Level denotes the number of levels or cells in the LUT cascade, the column Slice denotes the number of occupied slices, the column Memory denotes the amount of memory required, and the column F_clk denotes the maximum clock frequency. The column tco denotes the maximum clock-to-output propagation delay. (It is the maximum time required to obtain a valid output at the output pin that is fed by a register after a clock signal transition on an input pin that clocks the register). The column tpd denotes the maximum propagation time from the inputs to the outputs. The column Th. denotes the maximum throughput. Since the LPM address generator has 9 outputs, it is calculated as:

$$
\text { Th. }=9 \cdot \text { F_clk. }
$$

For Reg-Gates, Delay denotes the maximum delay from the input to the output and is equal to tpd. For multiple LUT cascade realizations and Xilinx's TCAM, Delay denotes the total delay, and is calculated by:

$$
\text { Delay }=\frac{1000 \cdot \text { Level }}{\text { F_clk }}+t c o,
$$

where 1000 is a unit conversion factor.

Consider the area occupied by the various realizations. From the Spartan-3 family architecture (Spartan-3 FPGA data sheet 2005), we can see that the area of one BRAM is at least the area of 16 slices (a slice consists of two "4-input LUTs", two flip-flops, and miscellaneous multiplexers).

An alternative estimate shows that the area of one BRAM is equivalent to that of 96 slices, as follows. In the Xilinx Virtex-II FPGA, one "4-input LUT" occupies approximately the same area as 96 bits of BRAM (also containing $18 \mathrm{~K}$ bits) (Sproull et al. 2005). Note that both "4-input LUTs" and BRAMs of the Virtex-II FPGA are similar to those of the Spartan-3 FPGA. Thus, 
Table 7. Comparisons of FPGA implementations of the LPM address generator.

\begin{tabular}{l|c|c|c|c|r|r|r|r|r|r}
\hline Design & Level & Slice & $\begin{array}{r}\text { Memory } \\
(\text { BRAM })\end{array}$ & $\begin{array}{r}\text { F_clk } \\
(\mathrm{MHz})\end{array}$ & $\begin{array}{r}\text { tco/tpd } \\
(\mathrm{ns})\end{array}$ & $\begin{array}{r}\text { Th. } \\
(\mathrm{Mbps})\end{array}$ & $\begin{array}{r}\text { Area } \\
(\text { slice })\end{array}$ & $\begin{array}{r}\text { Th./Area } \\
\left(\frac{\text { Mbps }}{\text { slice }}\right)\end{array}$ & $\begin{array}{r}\text { Delay } \\
(\mathrm{ns})\end{array}$ & $\begin{array}{r}\text { Area-Delay } \\
(\text { slice- } \mu \mathrm{s})\end{array}$ \\
\hline$r 6 p 11$ & 6 & 178 & 48 & 103.89 & $\begin{array}{r}24.89 \\
(\mathrm{tco})\end{array}$ & 935 & 4786 & 0.195 & 82.64 & 395.53 \\
\hline$r 7 p 11$ & 7 & 116 & 28 & 113.77 & $\begin{array}{r}23.46 \\
(\mathrm{tco})\end{array}$ & 1024 & 2804 & 0.365 & 84.99 & 238.31 \\
\hline$r 8 p 11$ & 8 & 69 & 16 & 139.93 & $\begin{array}{r}20.91 \\
(\mathrm{tco})\end{array}$ & $\begin{array}{r}\mathbf{1 2 5 9} \\
(\text { best })\end{array}$ & 1605 & 0.785 & 79.57 & 127.71 \\
\hline$r 9 p 11$ & 12 & 99 & 12 & 139.08 & $\begin{array}{r}13.72 \\
(\mathrm{tco})\end{array}$ & 1252 & $\begin{array}{r}\mathbf{1 2 5 1} \\
(\text { best })\end{array}$ & $\begin{array}{r}\mathbf{1 . 0 0 1} \\
(\text { best })\end{array}$ & 100.00 & $\begin{array}{r}\mathbf{1 2 5 . 1 0} \\
(\text { best })\end{array}$ \\
\hline $\begin{array}{l}\text { Xilinx's } \\
\text { TCAM }\end{array}$ & 1 & 8590 & & 22.52 & $\begin{array}{r}13.48 \\
(\mathrm{tco})\end{array}$ & 203 & 8590 & 0.024 & $\begin{array}{r}\mathbf{5 7 . 8 8} \\
(\text { best })\end{array}$ & 497.23 \\
\hline $\begin{array}{l}\text { Reg- } \\
\text { Gates }\end{array}$ & & 27646 & & & $\begin{array}{r}58.67 \\
(\mathrm{tpd})\end{array}$ & & 27646 & & 58.67 & 1621.99 \\
\hline
\end{tabular}

${ }^{a}$ We assume that the area for one BRAM is equivalent to the area of 96 slices.

we can deduce that one BRAM of the Spartan-3 FPGA occupies about the same area as $192(=18 \times 1024 / 96)$ "4-input LUTs". If we view one "4-input LUT" as approximately one-half a slice according to our discussion in the previous paragraph, we conclude that one BRAM has about the same area as $96(=192 / 2)$ slices. Thus, two estimates of the area for one BRAM, 16 and 96 slices are quite different. For this analysis, a worst case of 96 slices/BRAM was used.

In Table 7, the column Area denotes the equivalent utilized area, where the area for one BRAM is equivalent to the area for 96 slices. The column Th./Area denotes the efficiency of throughput per area for one slice. The column Area-Delay denotes the area-delay product. The value denoted by best shows the best result.

Xilinx's TCAM has the smallest delay, but requires many slices. Reg-Gates has almost the same delay as Xilinx's TCAM, but requires about three times as many slices as Xilinx's TCAM. Note that Reg-Gates requires no clock pulses in the LPM address generation operation, while the others are sequential circuits that require clock pulses. Since the delay of Reg-Gates is $58.67 \mathrm{~ns}$, the equivalent throughput is $(1000 / 58.67) \times 9=153(\mathrm{Mbps})$, which is lower than all others.

All multiple LUT cascade realizations have higher throughput, smaller area, higher throughput/area, and are more efficient in terms of area-delay than Xilinx's TCAM. $r 8 p 11$ has the highest throughput and the smallest delay among all multiple LUT cascade realizations, but is slightly less efficient in terms of area-delay than $r 9 p 11$. $r 9 p 11$ has the smallest area, the highest throughput/area, and the highest efficiency in terms of area-delay among all realizations. Although $r 8 p 11$ has almost the same area-delay as $r 9 p 11$, its area is $28 \%$ more larger than that of $r 9 p 11$. Hence, $r 9 p 11$ is the best multiple LUT cascade realization since it has 5.17 times more throughput, 40.71 times more throughput/area, and is 2.97 times more efficient in terms of area-delay product than Xilinx's TCAM, while the area is only $15 \%$ of Xilinx's TCAM. 
Table 8. Memory-Delay for multiple LUT cascade realizations.

\begin{tabular}{l|c|c|c|c}
\hline Design & $r 6 p 11$ & $r 7 p 11$ & $r 8 p 11$ & $r 9 p 11$ \\
\hline Area-Delay $($ slice- $\mu$ s $)$ & 395.53 & 238.31 & 127.71 & $\mathbf{1 2 5 . 1 0}$ \\
\hline Memory-Delay $($ BRAM- $\mu$ s) & 3.97 & 2.38 & 1.27 & $\mathbf{1 . 2 0}$ \\
\hline
\end{tabular}

\section{The optimum configuration of the multiple LUT cascade}

Firstly, consider the relation between the required memory size and the total area. As can be seen from Table 7, for $r 6 p 11$, which has the most complicated encoder, the memory required occupies $96.3 \%\left(=\frac{48 \times 96}{4786}\right)$ of the total area. For $r 9 p 11$, the memory required occupies $92.1 \%\left(=\frac{12 \times 96}{1251}\right)$ of the total area. Note that $r 9 p 11$ has the smallest proportion of the area for memory to the total area among all the multiple LUT cascade realizations. Thus, the memory consumes no less than $92 \%$ of the total area. In addition, as shown in Table 7, the size of Memory is approximately proportional to the Area. Hence, we can assume that the multiple LUT cascade realization with the smallest memory size corresponds to that with the smallest total area. Secondly, consider the relation between Area-Delay and Memory-Delay product. As shown in Table 8, $r 9 p 11$ has both the smallest Area-Delay and the smallest Memory-Delay among all multiple LUT cascade realizations. Note that the value of Memory-Delay is approximately proportional to the Area-Delay. Thus, we can assume that the realization with the smallest Memory-Delay corresponds to that with the smallest Area-Delay. Therefore, we can use the total size of memory required instead of the total area, and Memory-Delay instead of Area-Delay to find the optimum multiple LUT cascade realization. Doing this allows a formal analysis, as shown in the next section.

\subsection{Total size of memory}

Consider the multiple LUT cascade implementation of an $n$-input LPM address generator that stores $k$ prefix vectors. Let each cell be realized as a reconfigurable memory with $m$ bits. For the implementations discussed previously in this paper, this memory is a BRAM of the Spartan-3 FPGA, where $m=18,432$ bits. Each cell in the LUT cascade has $r$ outputs, where $r \leq\left\lceil\log _{2}(k+1)\right\rceil$. With $m$ bits stored in each memory and $r$ bits per word, $\frac{m}{r}$ words are stored in each LUT cell. Therefore, the number of address inputs for each LUT cell is $p(r)=\left\lfloor\log _{2} \frac{m}{r}\right\rfloor$. Note that $r \leq p(r)-1$. Let $M(r)$ be the total memory needed to implement the given LPM address generator. That is,

$$
M(r)=m s g,
$$


where $s=\left\lceil\frac{n-r}{p(r)-r}\right\rceil$ is the number of cells in each of the $g=\left\lceil\frac{k}{2^{r}-1}\right\rceil$ cascades that make up the multiple LUT cascade realization of the LPM address generator.

Theorem 6.1 $M(r)$ is a monotone decreasing function of $r$ for $r \leq p(r)-2$.

Since $M(r)$ is monotone decreasing for $r \leq p(r)-2$, to find the minimum $M(r)$, it is only necessary to find $M(r)$ for $r=p(r)-2$ and $r=p(r)-1$, an upper bound in $r$.

\subsection{Memory-delay product}

From Table 7, we observed that the delay in an $n$-input LPM address generator is given approximately as

$$
D=\frac{1000}{F_{-} c l k}(s+2)
$$

where $F \_c l k$ is the frequency of the clock in MHz. Let $M D(r)$ be the memorydelay product of the multiple LUT cascade realization of the address generator. Therefore,

$$
M D(r)=(m s g)\left(\frac{1000}{F_{\_} c l k}(s+2)\right),
$$

THEOREM 6.2 $M D(r)$ is a monotone decreasing function of $r$ for $r \leq p(r)-5$. Specially, when $p(r-1)=p(r), M D(r)$ is a monotone decreasing function of $r$ for $r \leq p(r)-3$.

Since $M D(r)$ is monotone decreasing for $r \leq p(r)-5$, to find the minimum $M D(r)$, it is only necessary to find $M D(r)$ for $r=p(r)-i$, where $i=1,2, \ldots$, 5 , or for five values of $r$. Specially, when $p(r-1)=p(r)$, to find the minimum $M D(r)$, we only need to consider three cases for $r=p(r)-i$, where $i=1,2$, and 3 .

\subsection{Optimum multiple LUT cascade for the BRAM containing $18 \mathrm{~K}$ bits, $16 K$ bits or $4 \mathrm{~K}$ bits}

In popular FPGAs, such as Xilinx's FPGAs or Altera's FPGAs, the sizes of BRAM are $18 \mathrm{~K}$ bits or $4 \mathrm{~K}$ bits. For other FPGAs, the size of BRAM can be $16 \mathrm{~K}$ bits. We consider these three types of BRAMs in the following discussion.

For 16K-bit BRAM, from $p(r)=\left\lfloor\log _{2}(16384 / r)\right\rfloor$, we have $p(r)=10$ for $r=9$ and $p(r)=11$ for $5 \leq r \leq 8$. Since $p(r-1)=p(r)=11$ for $5 \leq r \leq 8$, 
Table 9. The value of $r$ that makes $M D(r)$ minimum.

\begin{tabular}{c|l}
\hline Block_RAM size & The minimum Memory-Delay \\
\hline 18K bits & $r=r \_$max when $r \_m a x \leq 8$ \\
& $r=r \_o p t i m a l$ when $r \_$max $=9$ \\
\hline 4K bits & $r=r \_$max when $r \_m a x \leq 6$ \\
& $r=r_{-}$optimal when $r \_$max $=7$ \\
\hline 16K bits & $r=r \_$max for $r \leq 8$
\end{tabular}

$r \_\max$ is the maximum integer $r$ that satisfies both $r \leq p(r)-1$ and $r \leq\left\lceil\log _{2}(k+1)\right\rceil$, where $p(r)=\left\lfloor\log _{2} \frac{m}{r}\right\rfloor$ and $m$ denotes the size of a BRAM.

$r_{-}$optimal is $r$ that makes $s \cdot g \cdot(s+2)$ minimum, where $s=\left\lceil\frac{n-r}{p(r)-r}\right\rceil$ and $g=\left\lceil\frac{k}{2^{r}-1}\right\rceil$. For $m=18 \mathrm{~K}$-bit BRAM, r_optimal can be obtained by calculating values only for $r=p(r)-2=9$ and $r=p(r)-3=8$. For $m=4 \mathrm{~K}$-bit BRAM, $r_{-}$optimal can be obtained by calculating values only for $r=p(r)-2=7$ and $r=p(r)-3=6$.

from Theorem 6.2, $M D(r)$ decreases with $r$ when $1 \leq r \leq(11-3)=8$. Let $\zeta(r)=s \cdot g \cdot(s+2)$, where $s=\left\lceil\frac{n-r}{p(r)-r}\right\rceil$ and $g=\left\lceil\frac{k}{2^{r}-1}\right\rceil$. We can verify $\zeta(8)<\zeta(9)$ when $n>15$. In most applications, we can assume that $n>16$. Thus, we can conclude that $M D(r)$ is minimum when $r$ is maximum, where $r \leq 8$.

When the size of a BRAM is $m=18 \mathrm{~K}$ bits, from $p(r)=\left\lfloor\log _{2}(9 / r)\right\rfloor+11$, we have $p(r)=11$ for $5 \leq r \leq 9$. When $m=4 \mathrm{~K}$ bits, from $p(r)=\left\lfloor\log _{2}(8 / r)\right\rfloor+9$, we have $p(r)=9$ for $5 \leq r \leq 8$ and $p(r)=10$ for $r=4$. Thus, for both $m=18 \mathrm{~K}$-bit and $m=4 \mathrm{~K}$-bit, we have $p(r-1)=p(r)$ when $r \leq p(r)-5$. From Theorem 6.2, $M D(r)$ is minimum when $r=11-2=9$ or $r=11-3=8$ for 18K-bit BRAM, and $r=9-1=8, r=9-2=7$, or $r=9-3=6$ for $4 \mathrm{~K}$-bit BRAM. We can verify $\zeta(6)<\zeta(8)$ for $4 \mathrm{~K}$-bit BRAM when $n>14$. In most applications, we can assume that $n>16$. Thus, we only need to consider the case of $r=p(r)-2$. Depending on the values of $n$ and $k, M D(r)$ is minimum when $r=p(r)-2$ or $r=p(r)-3$. However, for an LPM address generator with fixed $n$ and $k$, we can easily obtain an $r$ that minimizes $\zeta(r)=s \cdot g \cdot(s+2)$ by calculating the values for $r=p(r)-2$ and $r=p(r)-3$.

Table 9 shows the values of $r$ that minimize $M D(r)$ for three types of BRAMs.

In Table 7, Area-Delay for $r 9 p 11$ and $r 8 p 11$ are nearly the same. Note that when $p=11$ and $n=32, \zeta(p-2)=0.30$ and $\zeta(p-3)=0.31$ are almost the same value.

For BRAM containing $18 \mathrm{~K}$ bits, Theorem 6.1 shows that the area for $r=9$ is smaller than for $r=8$. If $M D(r)$ for $r=9$ is almost the same as for $r=8$, then the multiple LUT cascade is optimum when $r=9$. Similar to $4 \mathrm{~K}$-bit BRAM, if $M D(r)$ for $r=7$ and $r=6$ are almost the same, then the multiple LUT cascade is optimum when $r=7$. The following example shows the design of an optimum multiple LUT cascade. 


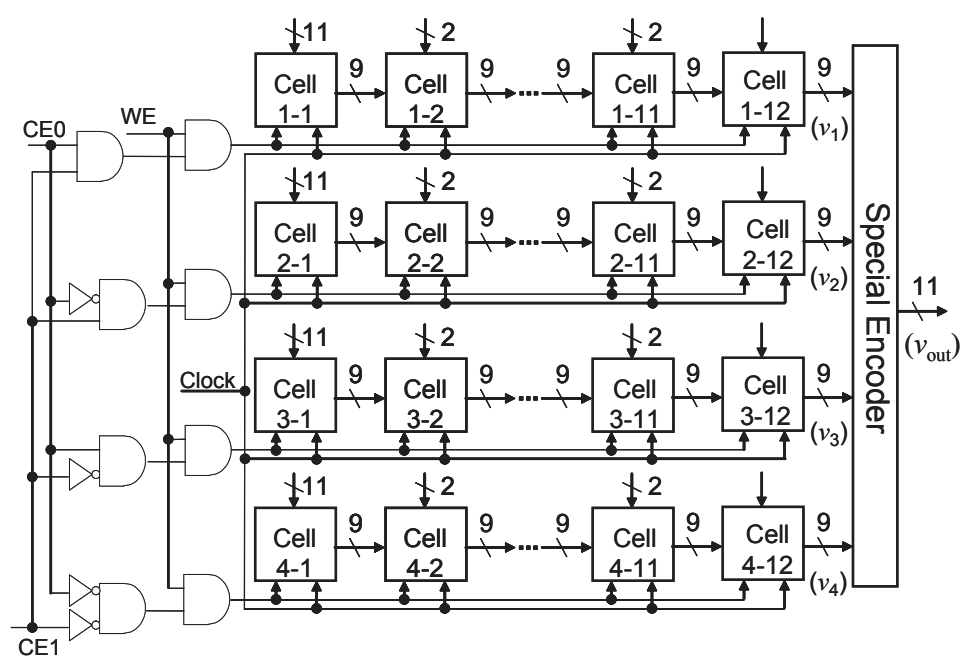

Figure 6. Optimum realization of $r 9 p 11 g 4$.

Example 6.3 Consider an LPM address generator with $n=32$ and $k=2040$ implemented on a Spartan-3 FPGA. Note that the size of a BRAM is $m=18 \mathrm{~K}$ bits. First, from $p(r)=\left\lfloor\log _{2}(9 / r)\right\rfloor+11$, we have $p(r)=11$ when $5 \leq r \leq 9$. To obtain the optimal Area-Delay realization, from Table $9, r$ can be 8 or 9 when $n=32$ and $k=2040$. Let $\zeta(r)=s \cdot g \cdot(s+2)$. We have $\zeta(9)=672$, and $\zeta(8)=640$. Note that $\zeta(9)$ is nearly the same as $\zeta(8)$. Since the area for $r=9$ is minimum from Theorem 6.1, the multiple LUT cascade is optimum when $p=11$ and $r=9$. In this case, a realization with $g=\left\lceil\frac{k}{2^{r}-1}\right\rceil=\left\lceil\frac{2044}{2^{9}-1}\right\rceil=4$ LUT cascades is optimum. Also, the number of levels is $\left\lceil\frac{n-r}{p-r}\right\rceil=\left\lceil\frac{32-9}{11-9}\right\rceil=12$, which shows that each LUT cascade consists of 12 cells. Finally, we need a special encoder. Let $v_{1}$ be the value of the outputs of the top LUT cascade, let $v_{2}$ be the value of the outputs of the second LUT cascade, let $v_{3}$ be the value of the outputs of the third LUT cascade, let $v_{4}$ be the value of the outputs of the fourth LUT cascade, and let $v_{\text {out }}$ be the value of the outputs of the special encoder. Then, we have the relation:

$$
v_{\text {out }}= \begin{cases}v_{4}+1533 & \text { if } v_{1}=v_{2}=v_{3}=0 \text { and } v_{4} \neq 0 \\ v_{3}+1022 & \text { if } v_{1}=v_{2}=0 \text { and } v_{3} \neq 0 \\ v_{2}+511 & \text { if } v_{1}=0 \text { and } v_{2} \neq 0 \\ v_{1} & \text { otherwise. }\end{cases}
$$

The optimum realization of $r 9 p 11 g 4$ is shown in Fig 6 .

We also implemented the LPM address generator with $n=32$ and $k=2040$ on Xilinx Spartan-3 FPGAs (XC3S4000-5). Table 10 shows that $r 9 p 11 g 4$ has 
Table 10. FPGA implementations of the LPM address generator with $n=32$ and $k=2040$.

\begin{tabular}{l|c|c|c|r|r|r|r|r|r|r}
\hline Design & Level & Slice & $\begin{array}{r}\text { Memory } \\
(\mathrm{BRAM})\end{array}$ & $\begin{array}{r}\text { F_clk } \\
(\mathrm{MHz})\end{array}$ & $\begin{array}{r}\text { tco } \\
(\mathrm{ns})\end{array}$ & $\begin{array}{r}\text { Th. } \\
(\mathrm{Mbps})\end{array}$ & $\begin{array}{r}\text { Area } \\
(\text { slice })\end{array}$ & $\begin{array}{r}\text { Th./Area } \\
\left(\frac{\text { Mbps }}{\text { slice }}\right)\end{array}$ & $\begin{array}{r}\text { Delay } \\
(\mathrm{ns})\end{array}$ & $\begin{array}{r}\text { Area-Delay } \\
(\text { slice- } \mu \mathrm{s})\end{array}$ \\
\hline$r 8 p 11 g 8$ & 8 & 299 & 64 & 111.20 & 26.00 & 1223 & 6443 & 0.190 & $\mathbf{9 7 . 9 4}$ & $\mathbf{6 3 1 . 0 4}$ \\
\hline$r 9 p 11 g 4$ & 12 & 241 & 48 & 111.33 & 23.00 & $\mathbf{1 2 2 5}$ & $\mathbf{4 8 4 9}$ & $\mathbf{0 . 2 5 3}$ & 130.79 & 634.19 \\
\hline
\end{tabular}

${ }^{a}$ We assume that the area for one BRAM is equivalent to the area of 96 slices.

$r 8 p 11 g 8$ denotes the FPGA implementation with $r=8, p=11$, and $g=8$.

$r 9 p 11 g 4$ denotes the FPGA implementation with $r=9, p=11$, and $g=4$

almost the same throughput and area-delay as $r 8 p 11 g 8$, but its area is only $75 \%$ of $r 8 p 11 g 8$. In addition, $r 9 p 11 g 4$ has higher throughput/area than that of $r 8 p 11 g 8$. Thus, $r 9 p 11 g 4$ is the optimum realization for the LPM address generator with $n=32$ and $k=2040$.

\section{Conclusions}

In this paper, we presented the multiple LUT cascade to realize LPM address generators. In addition, we discussed an approach to obtain the optimum configuration of multiple LUT cascade on FPGAs. Although we illustrated the design method for $n=32$ and $k=504 \sim 511$, it can be extended to other values of $n$ and $k$.

We implemented four LPM address generators (i.e. $r 6 p 11, r 7 p 11, r 8 p 11$, and $r 9 p 11$ ) on the Xilinx Spartan-3 FPGA (XC3S4000-5) by using the multiple LUT cascade. For comparison, on the same type of FPGA, we also implemented Xilinx's proprietary TCAM and Reg-Gates, an approach proposed by us as a likely solution to the LPM problem. Xilinx's TCAM has the smallest delay, but requires many slices. Reg-Gates has almost the same delay as Xilinx's TCAM, but requires the largest area, and requires about three times as many slices as Xilinx's TCAM. All multiple LUT cascade realizations have higher throughput, smaller area, higher throughput/area and more efficient in terms of area-delay product than Xilinx's TCAM.

\section{ACKNOWLEDGMENTS}

This research is partly supported by a Grant-in-Aid for Scientific Research from JSPS, MEXT, a grant from Kitakyushu Area Innovative Cluster Project, and by an NSA contract. 


\section{REFERENCES}

K. Pagiamtzis and A. Sheikholeslami, "Content-addressable memory (CAM) circuits and architectures: A tutorial and survey," IEEE Journal of SolidState Circuits, Vol. 41, No. 3, 2006, pp. 712-727.

H. Song and J. W. Lockwood, "Efficient packet classification for network intrusion detection using FPGA," Proc. ACM/SIGDA 13th International Symposium on Field Programmable Gate Arrays, 2005, pp. 238 - 245.

P. C. Wang, C. T. Chan, R. C. Chen, and H. Y. Chang, "An efficient ternary CAMs entry-reduction algorithm for IP forwarding engine," IEE Proceedings-Communications Vol. 152, 2005, pp. 172-176.

S. Kasnavi, V. C. Gaudet, P. Berube, and J. N. Amaral, "A novel hardwarebased longest prefix matching scheme for TCAMs," Proc. IEEE International Symposium on Circuits and Systems, 2005, pp. 3339 - 3342.

Renesas Technology Inc.: 9 M/18 M-bit Full Ternary CAM, Datasheet, February 2005.

P. Gupta, S. Lin, and N. McKeown, "Routing lookups in hardware at memory access speeds," Proc. IEEE INFOCOM, 1998, pp. 1241-1247.

S. Dharmapurikar, P. Krishnamurthy, and D. Taylor, "Longest prefix matching using Bloom filters," Proc. ACM SIGCOMM, 2003, pp. 201-212.

T. Sasao, and J.T. Butler, "Implementation of multiple-valued CAM functions by LUT cascades," Proc. IEEE International Symposium on Multiple-Valued Logic, May 2006, CD-ROM.

H. Qin, T. Sasao, and J.T. Butler, "Implementation of LPM address generators on FPGAs," Proc. International Workshop on Applied Reconfigurable Computing (ARC2006), March 2006, pp 170-181.

F. Shafai, K.J. Schultz, G.F.R. Gibson, A.G. Bluschke, D.E. Somppi, "Fully parallel 30-MHz, 2.5-Mb CAM," IEEE Journal of Solid-State Circuits, Vol. 33, No. 11, 1998, pp. 1690-1696.

T. Sasao, "Analysis and synthesis of weighted-sum functions," IEEE Trans. on Computer-Aided Design of Integrated Circuits and Systems, Vol. 25, No. 5, 2006, pp. 789-796.

Website of Xilinx is available at http://www.xilinx.com

Xilinx, Inc., "Spartan-3 FPGA family: Complete data sheet," DS099, Aug. 19, 2005.

T. Sproull, G. Brebner, C. Neely, "Mutable codesign for embedded protocol processing," Proc. IEEE 15th International Conference on Field Programmable Logic and Applications, 2005, pp. 51-56. 